\title{
A Cell-Permeable Biscyclooctyne As a Novel Probe for the Identification of Protein Sulfenic Acids
}

\author{
David J. McGarry, ${ }^{\dagger}$ Maria M. Shchepinova, ${ }^{\ddagger}$ Sergio Lilla, ${ }^{\dagger}$ Richard C. Hartley, ${ }^{\dagger}$ and Michael F. Olson ${ }^{*},, \S$ \\ †Cancer Research UK Beatson Institute, Garscube Estate, Switchback Road, Glasgow G61 1BD, United Kingdom \\ ${ }^{*}$ WestCHEM School of Chemistry, University of Glasgow, Glasgow G12 8QQ, United Kingdom \\ ${ }^{\S}$ Institute of Cancer Sciences, University of Glasgow, Glasgow G12 8QQ United Kingdom
}

\section{Supporting Information}

\begin{abstract}
Reactive oxygen species act as important second messengers in cell signaling and homeostasis through the oxidation of protein thiols. However, the dynamic nature of protein oxidation and the lack of sensitivity of existing molecular probes have hindered our understanding of such reactions; therefore, new tools are required to address these challenges. We designed a bifunctional variant of the strained bicyclo[6.1.0] nonyne (BCN-E-BCN) that enables the tagging of intracellular protein sulfenic acids for biorthogonal copper-free click chemistry. In validation studies, $\mathrm{BCN}-\mathrm{E}-\mathrm{BCN}$ binds the sulfenylated form of the actin-severing protein cofilin, while mutation of the cognate cysteine residues abrogates its binding. $\mathrm{BCN}-\mathrm{E}-\mathrm{BCN}$ is cell permeable and reacts rapidly with cysteine sulfenic acids in cultured cells. Using different azide-tagged conjugates, we demonstrate that BCN-E-BCN can be used in

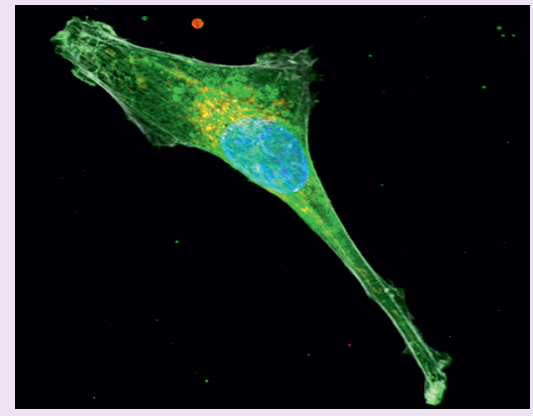
various applications for the detection of sulfenylated proteins. Remarkably, cycloaddition of an azide-tagged fluorophore to BCN-E-BCN labeled proteins produced in vivo can be visualized by fluorescence microscopy to reveal their localization. These findings demonstrate a novel and multifaceted approach to the detection and trapping of sulfenic acids.
\end{abstract}

$\mathrm{P}$ rotein thiol (sulfhydryl) groups are important mediators of redox signaling. ${ }^{1}$ In response to increases in cellular oxidation, thiols act as nucleophiles to reduce reactive oxygen species (ROS) in order to maintain redox homeostasis. In general, thiol oxidation leads to the formation of disulfides and oxidative intermediates, leading to structural changes of the protein thiols. ${ }^{2}$ In response to ROS, free thiols become oxidized to form sulfenic acids (sulfenylation), unstable moieties that can contribute to the formation of post-translational modifications that may vary depending on microenvironmental conditions and the duration of the ROS generated. ${ }^{3}$ Sulfenic acids may undergo further oxidation to sulfinic and sulfonic acids, or react with other cysteines or glutathione to form disulfides or thiosulfinates, respectively. ${ }^{4}$ These structural changes are diverse in nature and may impact protein function, activity, and localization. As such, ROS has significant influence on cell functions, acting as a second messenger to promote a diverse number of signaling events and subsequent responses including proliferation and differentiation, ${ }^{1,5}$ hypoxia, ${ }^{6}$ ferroptosis, ${ }^{7}$ and autophagy. ${ }^{8}$ Furthermore, there are clear ROS signatures in the etiology of several diseases; ${ }^{9}$ therefore, identifying the proteins subject to ROS regulation is critical to understanding how oxidative stress can influence these pathways and biological processes.

Protein thiol sulfenylation is central to ROS physiology and pathophysiology as it is the first intermediate step in protein oxidation. As such, a number of methods have been developed to exploit the reactivity of the protein thiol group in order to "trap" proteins in their sulfenylated state to characterize the proteins susceptible to ROS modification. Typically, the use of 1,3-dicarbonyl-based probes, such as dimedone, has been employed to label sulfenylated proteins, with over 700 proteins having been identified to date. ${ }^{10,11}$ Although the use of dimedone and dimedone-like compounds is generally accepted, there are a number of limitations associated with these probes. Dimedone derivatives have slow rates of reaction toward sulfenic acids; in fact, there have been several recent efforts to overcome this problem by balancing the $\mathrm{p} K_{\mathrm{a}}$ and nucleophilicity of the enolates of beta-functionalized carbonyl compounds. ${ }^{12,13}$ There is also evidence suggesting that they may react with electrophilic sulfenamides. ${ }^{14}$ Furthermore, due to the limited range of applications with such probes, there is a lack of spatial and temporal data revealing the subcellular distribution of sulfenylated proteins. As a result, there is a need to develop better tools to overcome the challenges to examining this dynamic and labile redox modification.

Strained cycloalkynes, such as bicyclo[6.1.0]nonyne (BCN), show great promise as sulfenic acid traps as they react with sulfenic acids with rate constants that are several-hundred-fold greater than other sulfenic acid probes. ${ }^{15}$ Rather than acting similarly to dimedone-based probes that exploit the electrophilicity of sulfenic acids (Supporting Information Figure S1A), ${ }^{12,13}$ or to probes that use the weak nucleophilicity of this group, ${ }^{16}$ the

Received: August 25, 2016

Accepted: October 28, 2016

Published: October 28, 2016 


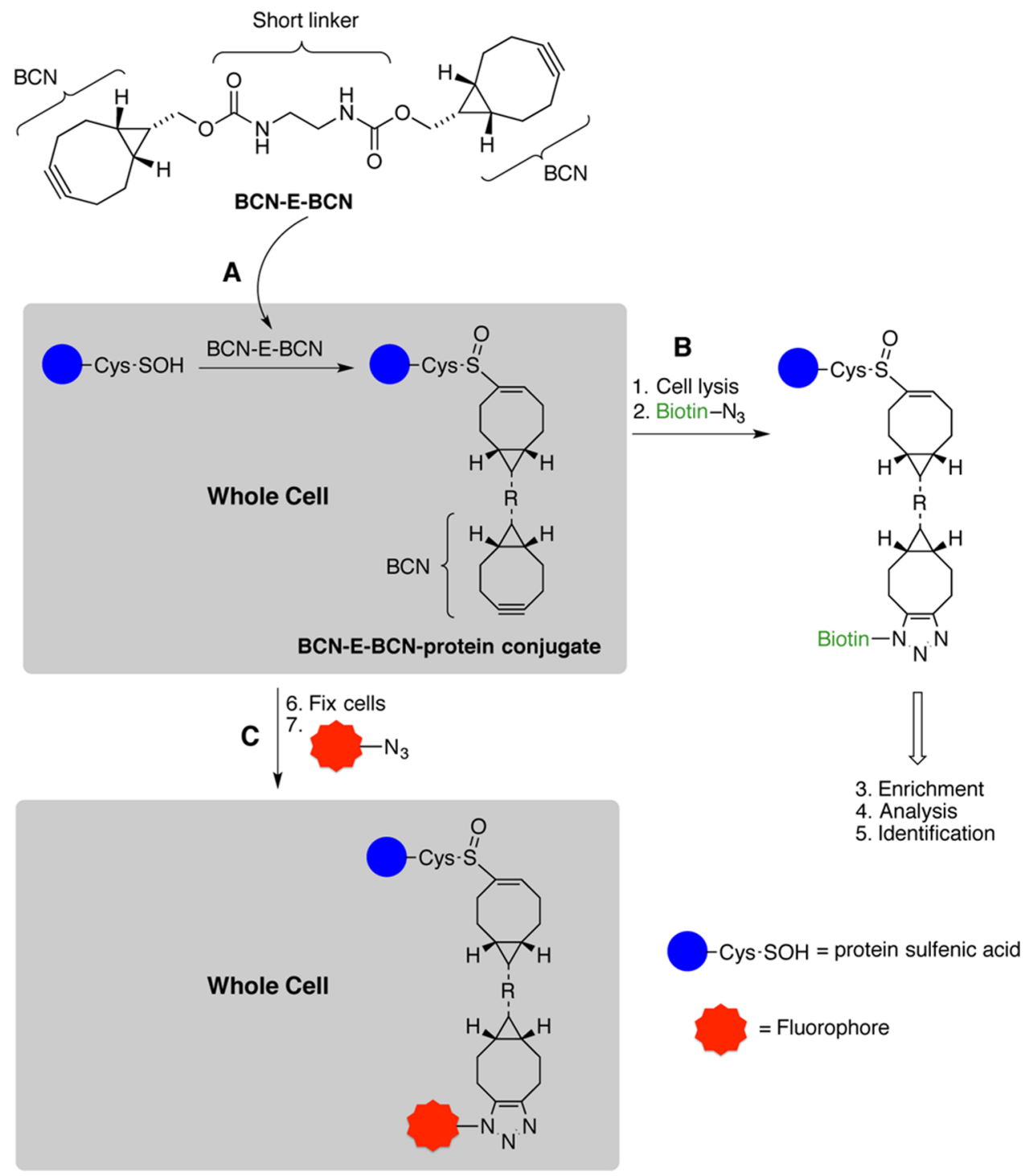

Figure 1. Design and application of BCN-E-BCN. (A) BCN-E-BCN (structure shown) permeates cells and reacts with endogenous protein cysteine sulfenic acids, thereby tagging them with a $\mathrm{BCN}$ for click chemistry. (B) Biotin can be attached using a copper-free click reaction between the $\mathrm{BCN}-\mathrm{E}-\mathrm{BCN}$-protein conjugate and an azide-tagged biotin, which could be used to assist protein or peptide enrichment and subsequent analysis. (C) In the same way, the BCN-E-BCN-protein conjugates can be labeled using an azide-tagged fluorophore to enable visualization of the subcellular distribution of protein sulfenic acids.

reaction involves a specific concerted syn addition to the alkyne (Supporting Information Figure S1B). In this study, we describe the development and validation of a novel cell-permeable bifunctional reagent, consisting of two linked $\mathrm{BCN}$ moieties (BCN-E-BCN), which makes use of the $\mathrm{BCN}$ reactivity for sulfenic acids to enable the detection of sulfenylated proteins.

$\mathrm{BCN}-\mathrm{E}-\mathrm{BCN}$ is comprised of two symmetrical strained cyclooctynes connected by a short ethylenediamine-derived linker, which allows protein sulfenic acids to be selectively tagged with a BCN group for copper-free click chemistry with azide-tagged reagents (Figure 1). The symmetrical design of the bifunctional probe allows for straightforward two-step synthesis from commercially available compounds ${ }^{17}$ (Supporting Information Figure S2) and exploits the most reactive and practical functionalities for sulfenic acid detection and click chemistry.

To validate the specificity of this probe, we incubated BCN-E-BCN with cofilin, a filamentous actin (F-actin) severing protein that has been shown to be oxidized by hydrogen peroxide $\left(\mathrm{H}_{2} \mathrm{O}_{2}\right){ }^{18}$ Recombinant human cofilin was first incubated with
$100 \mu \mathrm{M}$ BCN-E-BCN, followed by a copper-free click reaction with azide-PEG3-biotin. BCN-E-BCN tagging was characterized by Western blotting using fluorescently labeled streptavidin to detect consequent protein-biotin conjugates formed by this reaction. BCN-E-BCN tagged cofilin in vitro, which could be increased above spontaneously oxidized basal levels with increasing $\mathrm{H}_{2} \mathrm{O}_{2}$ concentrations (Figure $2 \mathrm{~A}$ ). We previously determined that cofilin oxidation by $\mathrm{H}_{2} \mathrm{O}_{2}$ occurs at cysteines 139 and 147 , resulting in reduced binding and severing of F-actin by cofilin. ${ }^{18}$ Mutation of these cysteines to alanine residues (C139/ 147A) decreased $\mathrm{H}_{2} \mathrm{O}_{2}$-induced $\mathrm{BCN}-\mathrm{E}-\mathrm{BCN}$ labeling in vitro (Figure 2B), indicating that $\mathrm{BCN}-\mathrm{E}-\mathrm{BCN}$ tags cofilin at these cysteine sulfenic acids. Mass spectrometry analysis of recombinant cofilin incubated with $\mathrm{BCN}-\mathrm{E}-\mathrm{BCN}$ verified that cysteine 139 was a $\mathrm{BCN}$-modified residue (Figure $2 \mathrm{C}$ ). In addition, we did not detect that BCN-E-BCN labeled the free thiol, sulfinic, or sulfonic forms of cofilin by mass spectrometry.

MDA MB 231 cells are triple negative human breast cancer cells that have been shown to have high basal levels of sulfenic 
A.

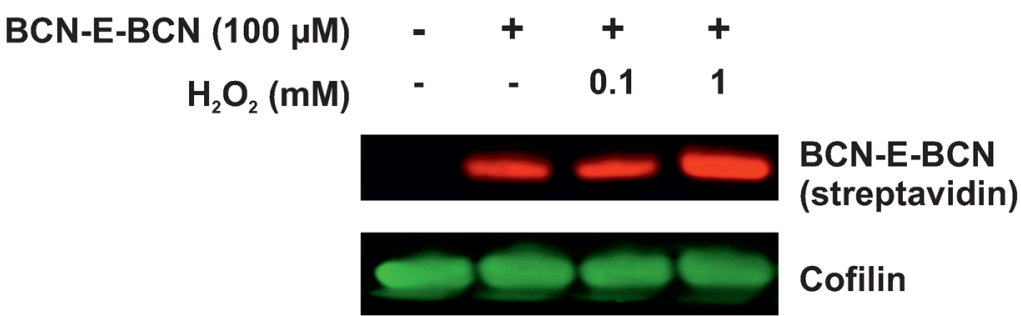

B.
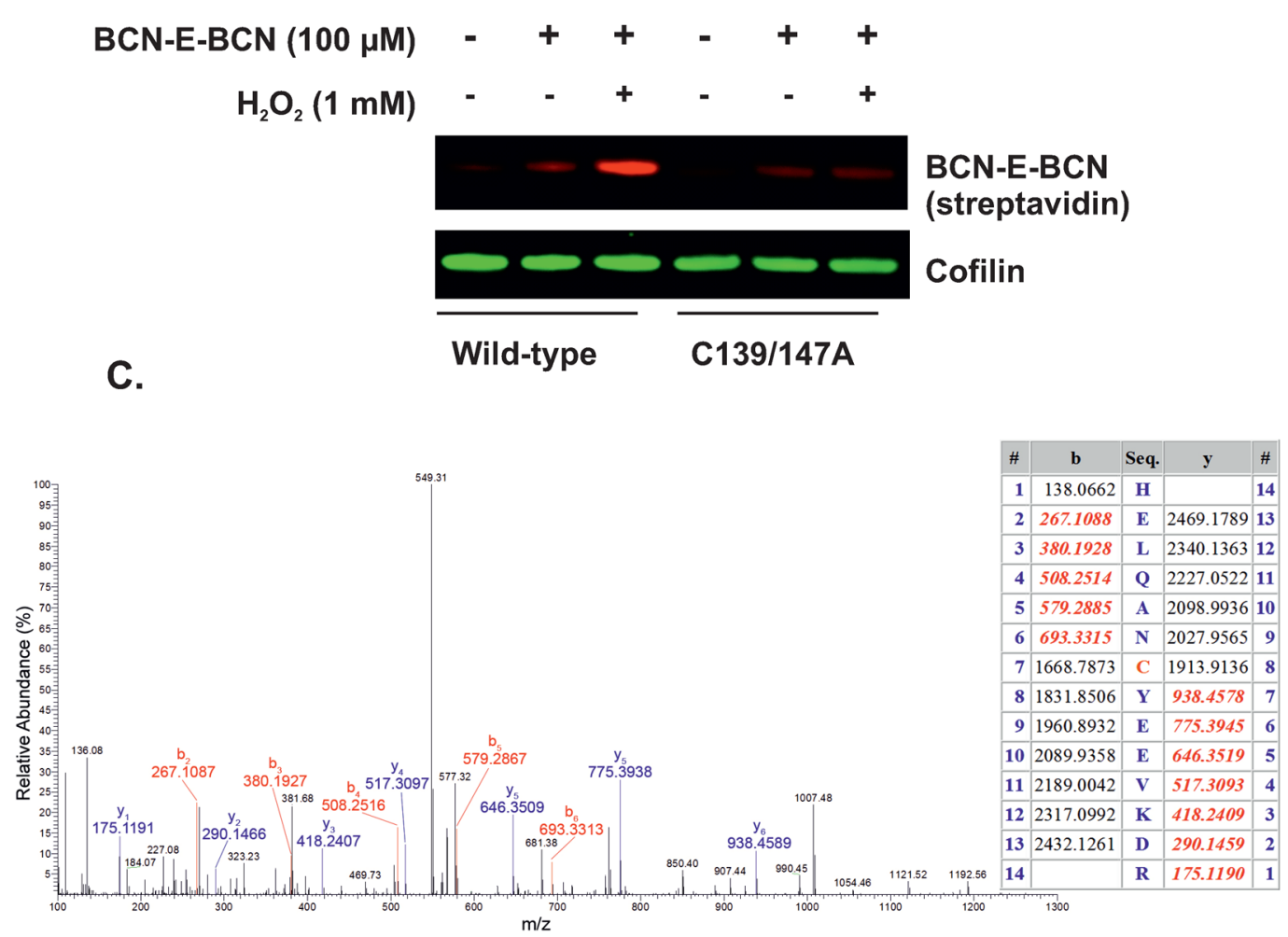

Figure 2. Validation of BCN-E-BCN sulfenic acid labeling using recombinant human cofilin protein. (A) Recombinant human cofilin (10 $\mu \mathrm{g})$ was oxidized with $\mathrm{H}_{2} \mathrm{O}_{2}(20 \mathrm{~min}, \mathrm{RT})$ before incubation with $100 \mu \mathrm{M} \mathrm{BCN}$-E-BCN. Azide-PEG3-biotin $(1 \mathrm{mM})$ was added for $1 \mathrm{~h}$ to drive the click reaction, and lysates were analyzed by Western blotting. BCN-E-BCN labeling was visualized using fluorophore-conjugated streptavidin and detected by infrared imaging. (B) Wild-type or C139/147A mutant human cofilin was oxidized in vitro with $1 \mathrm{mM} \mathrm{H}_{2} \mathrm{O}_{2}$ before being labeled with $100 \mu \mathrm{M} \mathrm{BCN}$-E-BCN and analyzed as described in A. (C) Positive ion MS/MS spectra and theoretical masses of $b$ and $y$ fragmentation series of a peptide (133-146) of human cofilin (P23528) modified with azide-PEG3-biotin BCN-E-BCN. The $b$ and $y$ ion series are indicated in blue and red, respectively.

acid formation. ${ }^{19}$ To test the reactivity of BCN-E-BCN in a whole cell system, we incubated cultured cells with varying concentrations of $\mathrm{BCN}-\mathrm{E}-\mathrm{BCN}$ for $30 \mathrm{~min}$ at $37^{\circ} \mathrm{C}$, followed by cell lysis and conjugation with azide-PEG3-biotin for detection. BCN-E-BCN was determined to be cell permeable and labeled sulfenic acids in a concentration-dependent manner (Figure 3A), with efficient labeling at concentrations lower than typically used for dimedone based probes. ${ }^{11}$ When compared to a commercially available biotinylated form of BCN (BCN-biotin), BCN-E-BCN demonstrated significantly greater efficacy in labeling sulfenylated proteins (Figure 3B). Treatment with $50 \mu \mathrm{M} \mathrm{H}_{2} \mathrm{O}_{2}$ induced a modest $50 \%$ increase in BCN-E-BCN labeling, as calculated by quantifying fluorophore-conjugated band intensities using an Odyssey CLx Imaging System (Figure 3C). To attenuate this increase and demonstrate that the labeling responds to changes in redox state, we incubated the reaction in the presence of the antioxidant $\mathrm{N}$-acetylcysteine (NAC; Figure 3C), which resulted in decreased $\mathrm{BCN}-\mathrm{E}-\mathrm{BCN}$ labeling. Furthermore, treatment with high $\mathrm{H}_{2} \mathrm{O}_{2}$ concentrations decreased $\mathrm{BCN}-\mathrm{E}-\mathrm{BCN}$ labeling and correlated with increased peroxiredoxin trioxide $\left(\mathrm{Prx}-\mathrm{O}_{3}\right)$ formation (Figure 3D), suggesting that proteins were oxidized beyond the single oxygen-containing sulfenic acid and were forming BCN nonreactive sulfinic and sulfonic acids at these concentrations. Collectively, these data are consistent with $\mathrm{BCN}-\mathrm{E}-\mathrm{BCN}$ being specific for the sulfenic acid form of thiol oxidation.

We next examined BCN-E-BCN tagging of a previously characterized sulfenylated candidate protein by immunoprecipitation from whole cell lysates. Glyceraldehyde 3-phosphate dehydrogenase $(\mathrm{GAPDH})$ is a key regulator of the glycolytic pathway and 
is susceptible to thiol oxidation. ${ }^{20-22}$ HEK 293 human embryonic kidney cell lysates were oxidized with $0.5 \mathrm{mM} \mathrm{H}_{2} \mathrm{O}_{2}$ before labeling with $250 \mu \mathrm{M}$ BCN-E-BCN. GAPDH was immunoprecipitated and $\mathrm{BCN}-\mathrm{E}-\mathrm{BCN}$ labeling determined by Western blotting. As shown in Figure 3E, BCN-E-BCN labeled GAPDH

A.

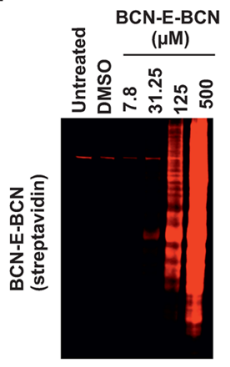

C.

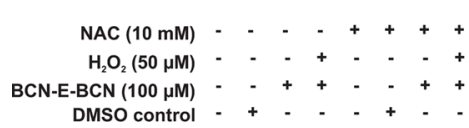

DMSO control

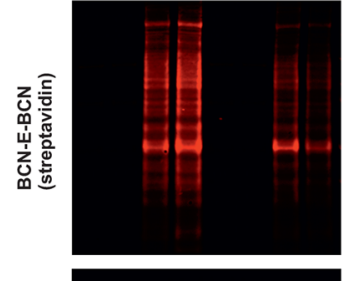

GAPDH

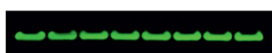

B.

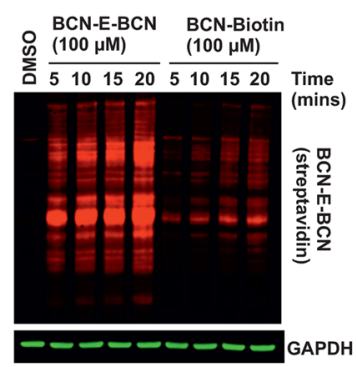

D.

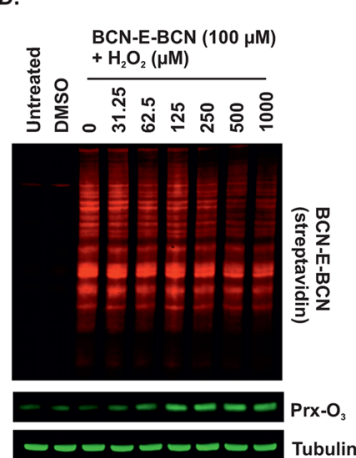

E.

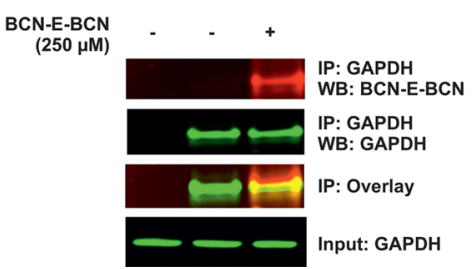

Figure 3. Biochemical properties of BCN-E-BCN in cultured cells. (A) MDA MB 231 human breast cancer cells were incubated with increasing concentrations of BCN-E-BCN for $30 \mathrm{~min}$ before being lysed and conjugated with $1 \mathrm{mM}$ azide-biotin using click chemistry. Data show Western blot analysis of total BCN-E-BCN labeling, which was visualized using fluorophore-conjugated streptavidin and detected by infrared imaging. (B) MDA MB 231 cells were incubated with either $100 \mu \mathrm{M}$ $\mathrm{BCN}-\mathrm{E}-\mathrm{BCN}$ or $100 \mu \mathrm{M} \mathrm{BCN}$-biotin and harvested at the time points indicated. BCN-E-BCN lysates were conjugated with $1 \mathrm{mM}$ azide-PEG3biotin before all lysates were resolved by Western blotting using fluorophore-conjugated streptavidin to detect BCN-E-BCN or BCNbiotin labeled proteins. GAPDH was used as a loading control. (C) MDA MB 231 cells were pretreated with $\mathrm{N}$-acetylcysteine (NAC: $10 \mathrm{mM}, 18 \mathrm{~h}$ ) before incubation with $\mathrm{H}_{2} \mathrm{O}_{2}$ for $20 \mathrm{~min}$. Cells were labeled with $100 \mu \mathrm{M}$ BCN-E-BCN and analyzed as described in A. (D) MDA MB 231 cells were incubated with increasing concentrations of $\mathrm{H}_{2} \mathrm{O}_{2}$ for $20 \mathrm{~min}$ before incubation with $100 \mu \mathrm{M}$ BCN-E-BCN for $30 \mathrm{~min}$. Cells were lysed, and BCN-E-BCN-protein conjugates were reacted with azidePEG3-biotin and analyzed as described in A. Protein oxidation to cysteine sulfonic acid was detected with antibody against peroxiredoxin sulfonic acid $\left(\operatorname{Prx}-\mathrm{O}_{3}\right)$, and $\beta$-tubulin was blotted as a loading control. (E) HEK 293 human embryonic kidney cell lysates were treated with $\mathrm{H}_{2} \mathrm{O}_{2}(500 \mu \mathrm{M}, 5 \mathrm{~min})$ and labeled with $250 \mu \mathrm{M}$ BCN-E-BCN before azide-biotin conjugation. GAPDH was immunoprecipitated from cells and the amount of BCN-E-BCN labeling was visualized using fluorophore-conjugated streptavidin and detected by infrared imaging. in HEK293 cell lysates, demonstrating the utility of this compound in identifying sulfenylated proteins from complex lysates.

To utilize the flexibility of the cyclooctyne ring in click chemistry reactions, we employed an azide-tagged fluorophore to label BCN-E-BCN-protein conjugates in fixed cells in order to visualize their subcellular localization. MDA MB 231 cells were fixed with $4 \%$ paraformaldehyde, tagged with $100 \mu \mathrm{M}$ BCN-E-BCN followed by Alexa Fluor 488 azide and analyzed by fluorescence microscopy. We were able to visualize the spatial distribution of $\mathrm{BCN}-\mathrm{E}-\mathrm{BCN}$ tagged proteins, with the majority being predominantly cytoplasmic, with some mitochondrial, plasma membrane and weak nuclear signals detected (Figure 4).

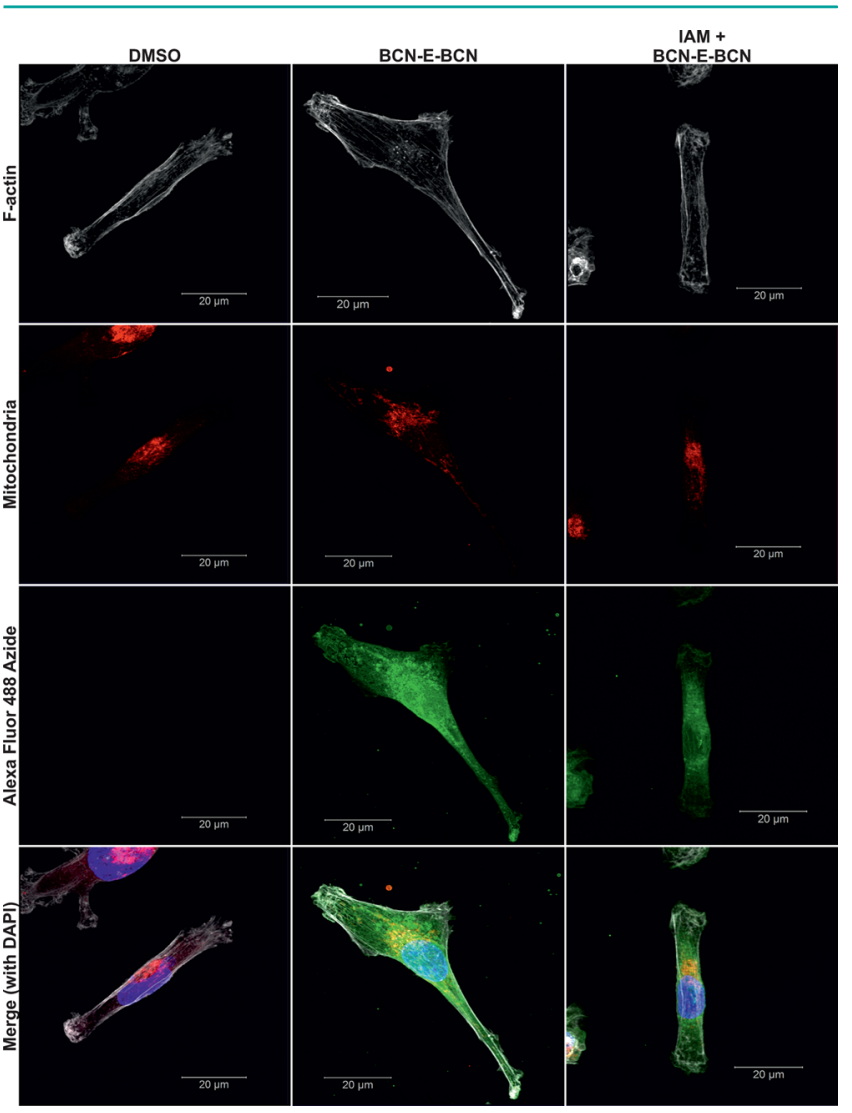

Figure 4. BCN-E-BCN enables visualization of sulfenylated proteins by fluorescence microscopy. MDA MB 231 human breast cancer cells were fixed with $4 \%$ paraformaldehyde, permeabilized for 5 min with $0.5 \%$ Triton, and incubated with $100 \mu \mathrm{M} \mathrm{BCN}-\mathrm{E}-\mathrm{BCN}$ for $30 \mathrm{~min}$. For iodoacetamide (IAM) treated samples, cells were pretreated with $100 \mu \mathrm{M}$ IAM (30 min, RT) prior to BCN-E-BCN incubation. Cells were washed in PBS and conjugated with $1 \mathrm{mM}$ Alexa fluor 488 azide for the detection of BCN-E-BCN labeled proteins. Cells were stained with an anti-Cox IV antibody (mitochondria), DAPI (nuclear DNA), and phalloidin (F-actin) as indicated.

It has been suggested that free thiols may undergo thiol-yne addition with cyclooctyne groups, such that pretreating proteins with iodoacetamide (IAM) would be expected to reduce nonspecific binding. ${ }^{23}$ However, we found that IAM pretreatment of MDA MB 231 cells did not markedly affect BCN-E-BCN labeling (Figure 4), consistent with the sulfenic acid specificity of the reaction and in line with previous findings. ${ }^{15}$ It should be noted that BCN should not be regarded as an electrophilic reagent for sulfenic acids but reacts via a concerted syn addition of the sulfenic acid to the alkyne: the $\mathrm{C}-\mathrm{S}$ bond and the $\mathrm{C}-\mathrm{H}$ bond are formed in the same step via a cyclic transition state, analogous 
to a pericyclic reaction (Supporting Information Figure S1B). This type of reactivity is not possible for protein thiols or sulfenamides, and our results indicate that electrophilic reaction with thiolates or thiyl radicals is unlikely to contribute to the observed $\mathrm{BCN}$-tagging of proteins under physiological conditions.

Protein sulfenylation has received much recent interest because of its dynamic and complex role in ROS mediated cell signaling. However, a major limitation is a lack of convenient tools to measure this post-translational modification. Existing reagents are compromised by low reaction rates for sulfenic acids or have limited utility in biochemical and cell biological applications. Here, we now report the development and characterization of a novel bifunctional cyclooctane compound, $\mathrm{BCN}-\mathrm{E}-\mathrm{BCN}$, which can rapidly label sulfenylated proteins in cultured cells and be used in a number of diverse applications. We demonstrate that BCN-E-BCN is cell permeable, has high reactivity compared to other $\mathrm{BCN}$ derivatives, and can facilitate a range of experimental approaches via copper-free click chemistry reactions that allow for labeling of $\mathrm{BCN}$-tagged proteins with a variety of readily detectable moieties.

\section{ASSOCIATED CONTENT}

\section{S Supporting Information}

The Supporting Information is available free of charge on the ACS Publications website at DOI: 10.1021/acschembio.6b00742.

Methods and Figures S1 and S2 (PDF)

\section{AUTHOR INFORMATION}

\section{Corresponding Author}

*E-mail: m.olson@beatson.gla.ac.uk.

\section{Notes}

The authors declare no competing financial interest.

\section{ACKNOWLEDGMENTS}

This project was sponsored by funding to M.F.O. from Cancer Research UK (A18276), the Medical Research Council (MR/M018776/1), and the Engineering and Physical Sciences Research Council (EP/N014642/1) and by funding to M.M.S. from the Engineering and Physical Sciences Research Council (EP/J500434/1).

\section{REFERENCES}

(1) Groitl, B., and Jakob, U. (2014) Thiol-based redox switches. Biochim. Biophys. Acta, Proteins Proteomics 1844, 1335-1343.

(2) Reczek, C. R., and Chandel, N. S. (2015) ROS-dependent signal transduction. Curr. Opin. Cell Biol. 33, 8-13.

(3) Jacob, C., Battaglia, E., Burkholz, T., Peng, D., Bagrel, D., and Montenarh, M. (2012) Control of oxidative posttranslational cysteine modifications: from intricate chemistry to widespread biological and medical applications. Chem. Res. Toxicol. 25, 588-604.

(4) Kettenhofen, N. J., and Wood, M. J. (2010) Formation, reactivity, and detection of protein sulfenic acids. Chem. Res. Toxicol. 23, 16331646.

(5) Rudzka, D. A., Cameron, J. M., and Olson, M. F. (2015) Reactive oxygen species and hydrogen peroxide generation in cell migration. Commun. Integr. Biol. 8, e1074360.

(6) Murphy, M. P. (2012) Modulating mitochondrial intracellular location as a redox signal. Sci. Signaling 5, pe39.

(7) Xie, Y., Hou, W., Song, X., Yu, Y., Huang, J., Sun, X., Kang, R., and Tang, D. (2016) Ferroptosis: process and function. Cell Death Differ. 23, 369-379.
(8) Filomeni, G., De Zio, D., and Cecconi, F. (2015) Oxidative stress and autophagy: the clash between damage and metabolic needs. Cell Death Differ. 22, 377-388.

(9) Holmstrom, K. M., and Finkel, T. (2014) Cellular mechanisms and physiological consequences of redox-dependent signalling. Nat. Rev. Mol. Cell Biol. 15, 411-421.

(10) Yang, J., Gupta, V., Tallman, K. A., Porter, N. A., Carroll, K. S., and Liebler, D. C. (2015) Global, in situ, site-specific analysis of protein Ssulfenylation. Nat. Protoc. 10, 1022-1037.

(11) Yang, J., Gupta, V., Carroll, K. S., and Liebler, D. C. (2014) Sitespecific mapping and quantification of protein S-sulphenylation in cells. Nat. Commun. 5, 4776.

(12) Qian, J., Wani, R., Klomsiri, C., Poole, L. B., Tsang, A. W., and Furdui, C. M. (2012) A simple and effective strategy for labeling cysteine sulfenic acid in proteins by utilization of beta-ketoesters as cleavable probes. Chem. Commun. (Cambridge, U. K.) 48, 4091-4093.

(13) Gupta, V., and Carroll, K. S. (2016) Profiling the Reactivity of Cyclic C-Nucleophiles towards Electrophilic Sulfur in Cysteine Sulfenic Acid. Chem. Sci. 7, 400-415.

(14) Shiau, T. P., Erlanson, D. A., and Gordon, E. M. (2006) Selective reduction of peptide isothiazolidin-3-ones. Org. Lett. 8, 5697-5699.

(15) Poole, T. H., Reisz, J. A., Zhao, W., Poole, L. B., Furdui, C. M., and King, S. B. (2014) Strained cycloalkynes as new protein sulfenic acid traps. J. Am. Chem. Soc. 136, 6167-6170.

(16) Devarie Baez, N. O., Reisz, J. A., and Furdui, C. M. (2015) Mass spectrometry in studies of protein thiol chemistry and signaling: opportunities and caveats. Free Radical Biol. Med. 80, 191-211.

(17) Dommerholt, J., Schmidt, S., Temming, R., Hendriks, L. J., Rutjes, F. P., van Hest, J. C., Lefeber, D. J., Friedl, P., and van Delft, F. L. (2010) Readily accessible bicyclononynes for bioorthogonal labeling and threedimensional imaging of living cells. Angew. Chem., Int. Ed. 49, 94229425.

(18) Cameron, J. M., Gabrielsen, M., Chim, Y. H., Munro, J., McGhee, E. J., Sumpton, D., Eaton, P., Anderson, K. I., Yin, H., and Olson, M. F. (2015) Polarized Cell Motility Induces Hydrogen Peroxide to Inhibit Cofilin via Cysteine Oxidation. Curr. Biol. 25, 1520-1525.

(19) Seo, Y. H., and Carroll, K. S. (2009) Profiling protein thiol oxidation in tumor cells using sulfenic acid-specific antibodies. Proc. Natl. Acad. Sci. U. S. A. 106, 16163-16168.

(20) McGarry, D. J., Chen, W., Chakravarty, P., Lamont, D. L., Wolf, C. R, and Henderson, C. J. (2015) Proteome-wide identification and quantification of S-glutathionylation targets in mouse liver. Biochem. J. $469,25-32$.

(21) Baty, J. W., Hampton, M. B., and Winterbourn, C. C. (2005) Proteomic detection of hydrogen peroxide-sensitive thiol proteins in Jurkat cells. Biochem. J. 389, 785-795.

(22) Peralta, D., Bronowska, A. K., Morgan, B., Doka, E., Van Laer, K., Nagy, P., Grater, F., and Dick, T. P. (2015) A proton relay enhances $\mathrm{H} 2 \mathrm{O} 2$ sensitivity of GAPDH to facilitate metabolic adaptation. Nat. Chem. Biol. 11, 156-163.

(23) van Geel, R., Pruijn, G. J., van Delft, F. L., and Boelens, W. C. (2012) Preventing thiol-yne addition improves the specificity of strainpromoted azide-alkyne cycloaddition. Bioconjugate Chem. 23, 392-398. 